\title{
First signatures of strong differential rotation in A-type stars ${ }^{\star}$
}

\author{
A. Reiners ${ }^{1}$ and F. Royer ${ }^{2,3}$ \\ 1 Hamburger Sternwarte, Universität Hamburg, Gojenbergsweg 112, 21029 Hamburg, Germany \\ e-mail: areiners@hs.uni-hamburg.de \\ 2 Observatoire de Genève, 51 chemin des Maillettes, 1290 Sauverny, Switzerland \\ e-mail: frederic.royer@obs.unige.ch \\ 3 GEPI, CNRS UMR 8111, Observatoire de Paris, 5 place Janssen, 92195 Meudon cedex, France
}

Received 8 August 2003 / Accepted 6 November 2003

\begin{abstract}
We reanalyzed high quality spectra of 158 stars of spectral types A0-F1 and $v \sin i$ between 60 and $150 \mathrm{~km} \mathrm{~s}^{-1}$. Using a Least Squares Deconvolution technique we extracted high $S / N$ broadening profiles and determined the loci of the Fourier transform zeros $q_{1}$ and $q_{2}$ where the $S / N$-ratio was high enough. The values of $v \sin i$ were redetermined and found to be consistent with the values derived by Royer et al. (2002). For 78 stars $q_{2}$ could be determined and the ratio $q_{2} / q_{1}$ was used as a shape parameter sensitive for solar-like differential rotation (Equator faster than Pole). 74 of the 78 stars have values of $q_{2} / q_{1}$ consistent with solid body rotation; in four of the 78 cases, values of $q_{2} / q_{1}$ are not consistent with rigid rotation. Although these stars may be binaries, none of the profiles shows signatures of a companion. The Fourier transforms do not indicate any distortions and the broadening profiles can be considered due to single objects. One of those candidates may be an extremely rapid rotator seen pole-on, but for the other three stars of spectral types as early as A6, differential rotation seems to be the most plausible explanation for the peculiar profiles.
\end{abstract}

Key words. stars: rotation - stars: early-type - stars: activity

\section{Introduction}

The substantial difference between photospheres of solar-type stars and A-type stars is the existence of a convective envelope. Due to the ionization of hydrogen the cooler late-type stars harbour convective envelopes where turbulent motions of the photospheric plasma can occur. Stars of spectral types earlier than about F2 have no or only very thin convective envelopes and properties of granular flows change fundamentally.

The generally accepted activity paradigm places the stellar dynamo believed to cause stellar activity at the boundary between the convective envelope and the radiative core. Differential rotation drives the dynamo action by winding up and amplifying the magnetic flux tubes. The interaction of magnetic fields, differential rotation and the convective envelopes are believed to be ultimately responsible for stellar activity.

In a series of publications an onset of convection was searched for. It is generally accepted that the onset of stellar activity occurs between spectral types A7 and F5 depending on the observational strategy. Wolff et al. (1986) studied C II and HeI emission and placed the onset of activity near

Send offprint requests to: A. Reiners,

e-mail: areiners@hs.uni-hamburg.de

* Based on observations collected at the European Southern Observatory, La Silla.
$B-V=0.28$, i.e. around spectral type F0. Schmitt (1997) concluded from X-ray data that coronal emission is universal in the spectral range A7 to G9 implying an onset of activity around spectral type A7. Hotter stars are expected to harbour shallow convective envelopes, these stars have higher convective velocities which peak at about A3 until convection disappears altogether at about A1 (Renzini et al. 1977). Gray \& Nagel (1989) directly searched for the onset of convection analyzing line bisectors of slowly rotating stars. In their targets the Doppler-shift distribution of the granulation dominates the broadening of spectral lines and a bisector reversal was found around spectral type F0. Stronger asymmetries were found in the stars at the hot side of the boundary indicating higher convective velocities.

Although stellar activity is not observed in early A-type stars, it is not clear whether differential rotation may take place in early-type stars. The absence of activity may simply reflect inefficient coupling of surface magnetic fields and the lacking interface between the radiative core and a convective envelope. There is no reason to believe that rapidly rotating A-stars should rotate rigidly. In case of the late-type Sun we know that the surface rotation law can be approximated by

$\Omega(l)=\Omega_{\text {Equator }}\left(1-\alpha \sin ^{2} l\right)$,

with $l$ the latitude and $\alpha_{\odot} \sim 0.2$ as derived from Sun spots. In the solar case the Equator rotates about $20 \%$ faster than the 
Poles. Gray (1977) searched for differential rotation in line profiles of six A-stars finding no indications of differential rotation within his error bars.

Also using line profiles, Reiners \& Schmitt (2003) found signatures of differential rotation in a sample of F-type stars. The earliest object in their sample indicating differential rotation is of spectral type F0IV/V. Applying the method used by Reiners \& Schmitt (2003), we search for signatures of differential rotation in a large sample of A-star spectra. The results are presented in the following.

\section{Observations and data analysis}

The spectra were observed with the ECHELEC spectrograph (ESO/La Silla) and are part of a larger sample collected in the framework of an ESO Key Programme. These observations were aimed at the determination of fundamental parameters of early-type stars observed by HIPPARCOS (Gerbaldi \& Mayor 1989). The total sample is described by Grenier et al. (1999) who measured radial velocities, and Royer et al. (2002, hereafter RGFG) who derived rotational velocities from these spectra. The observed spectral range spans from 4210 to $4500 \AA$. The linear dispersion is about $3.1 \AA \mathrm{mm}^{-1}$, the slit width of $320 \mu \mathrm{m}$ corresponds to $1{ }^{\prime \prime} 52$ on the sky, and the resolving power is about 28000 .

To search for the spectral signatures of stellar rotation laws, broadening profiles were derived by applying a Least Squares Deconvolution process (LSD). After constructing a $\delta$-template comprising the strongest 150 lines taken from the Vienna Atomic Line Database (Kupka et al. 1999) and according to stellar temperature, a first-guess broadening profile was deconvolved using each pixel as a free parameter in the fit. Since theoretical line depths match the observational ones poorly, the equivalent widths of the incorporated lines were optimized in a second step while leaving the broadening profile fixed. During a few iterations the broadening profile and the equivalent widths were optimized. Using this technique the spectral lines are effectively deblended, the information contained in every spectral line is used and the signal-to-noise ratio is significantly enhanced. Consistency of the fit is checked by comparing theoretical line depths to the derived ones.

Following Reiners \& Schmitt (2002) we Fourier transformed the broadening functions and measured the position of the first and second zeros $\left(q_{1}, q_{2}\right)$. The ratio $q_{2} / q_{1}$ is a robust observable for the shape of a rotational broadening function and a direct indicator for solar-like differential rotation with the Equator rotating faster than the Pole (cp. Reiners \& Schmitt 2002). We measured the ratio $q_{2} / q_{1}$ for all stars the LSD procedure yielded a stable and symmetric broadening function for.

The spectral quality used in this analysis in principle was sufficient to follow the Fourier transformed broadening functions to the second zero $q_{2}$ in stars with projected rotational velocities in the range $60 \mathrm{~km} \mathrm{~s}^{-1}<v \sin i<150 \mathrm{~km} \mathrm{~s}^{-1}$.

\section{Results}

The outlined method was applied to the spectra of 158 stars of spectral types A0-F1. The rotational velocity $v \sin i$ was

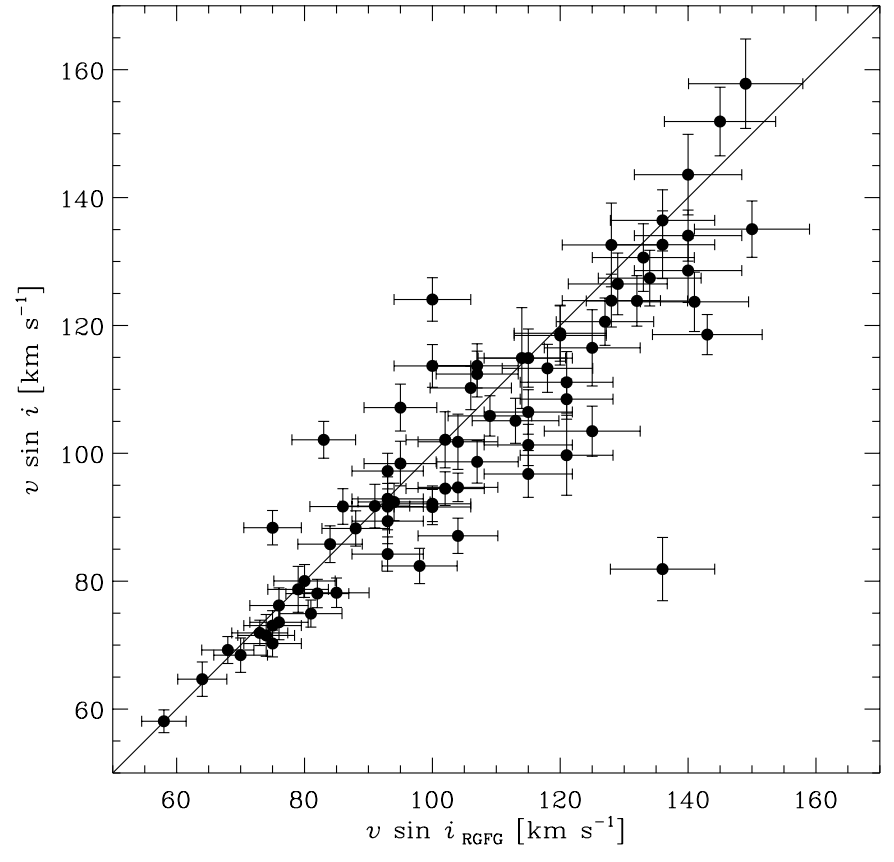

Fig. 1. Comparison of the $v \sin i$ values with the ones derived by RGFG. The solid line is the one-to-one relation, and the linear regression between both data sets is given in Eq. (2).

derived from the first zero $q_{1}$. For 78 of our sample stars the ratio $q_{2} / q_{1}$ could be determined. For the disregarded 80 stars, either data quality was insufficient or the derived broadening function showed obvious peculiarities probably due to binarity.

The $v \sin i$ values are compared with the results from RGFG in Fig. 1, for the subsample of 78 stars. RGFG also determined their values of $v \sin i$ from $q_{1}$ but used selected absorption lines while an "overall" broadening profile was deconvolved here. The consistency of both scales is robustly estimated using GaussFit (Jefferys et al. 1998a,b). The resulting linear relation is

$v \sin i=0.99 \pm 0.05 v \sin i_{\mathrm{RGFG}}-1.6 \pm 4.6$,

both scales are in good agreement. The systematic differences occuring due to the different ways obtaining the broadening profiles can be neglected in our sample and should not be discussed here.

We measured the second zeros of the Fourier transformed broadening profiles to calculate the ratios $q_{2} / q_{1}$. The results are plotted in Fig. 2, typical errors are of the order of $\Delta q_{2} / q_{1} \approx$ 0.1 . A rigid rotator is expected to yield a value of $q_{2} / q_{1}$ between 1.72 and 1.83 assuming a linear limb darkening law (indicated by dashed lines in Fig. 2). For the stars of our sample linear limb darkening coefficients between 0.5 and 0.75 are expected during their time on the Main Sequence (Claret 1998). Assuming a limb darkening parameter of $\epsilon=0.6$ rigid rotation would yield $q_{2} / q_{1}=1.76$ (solid line in Fig. 2). The results that are consistent with a value of $q_{2} / q_{1}=1.76$ within the error bars are indicated by small crosses in Fig. 2, for the sake of readability no errors are plotted for them. The second zero $q_{2}$ can only be determined in spectra where the signal exceeds the noise level beyond $q_{2}$, i.e. when a second sidelobe is detectable. In our case the amplitude of the second sidelobe is 
Table 1. Derived values of $v \sin i$ and $q_{2} / q_{2}$ for the four stars with values of $q_{2} / q_{1}$ significantly smaller than 1.76 . Also given are strength of differential rotation in terms of $\alpha$ (cp. Eq. (1)) and required values of equatorial velocities $v_{\mathrm{e} \text {,rigid }}$ and inclination angles $i$ if the value of $q_{2} / q_{1}$ is explained by rapid rotation seen pole-on (Sect.3.2). Distances in pc, ROSAT X-ray luminosities and $v$ sin $i$ derived in RGFG are given in Cols. 8-10.

\begin{tabular}{|c|c|c|c|c|c|c|c|c|c|}
\hline HD & Type & $\begin{array}{c}v \sin i \\
{\left[\mathrm{~km} \mathrm{~s}^{-1}\right]}\end{array}$ & $q_{2} / q_{1}$ & $\alpha$ & $\begin{array}{c}v_{\mathrm{e}, \text { rigid }} \\
{\left[\mathrm{km} \mathrm{s}^{-1}\right]}\end{array}$ & $i$ & $\begin{array}{r}d \\
{[\mathrm{pc}]}\end{array}$ & $\begin{array}{c}L_{\mathrm{X}} \\
{\left[\mathrm{W} \mathrm{m}^{-2}\right]}\end{array}$ & $\begin{array}{c}v \sin i \\
(\mathrm{RGFG})\end{array}$ \\
\hline 6869 & A9V & $100 \pm 6$ & $1.55 \pm 0.08$ & $0.28 \pm 0.10$ & $(460)$ & $\left(13^{\circ}\right)$ & 87 & 570 & 121 \\
\hline 60555 & A6V & $115 \pm 7$ & $1.54 \pm 0.07$ & $0.29 \pm 0.08$ & $(470)$ & $\left(14^{\circ}\right)$ & 134 & & 114 \\
\hline 109238 & F0IV/V & $103 \pm 4$ & $1.51 \pm 0.11$ & $0.32 \pm 0.13$ & $(500)$ & $\left(13^{\circ}\right)$ & 133 & & 125 \\
\hline 44892 & A9/F0IV & $152 \pm 5$ & $1.64 \pm 0.10$ & $0.16 \pm 0.16$ & 400 & $22^{\circ}$ & 160 & & 145 \\
\hline
\end{tabular}

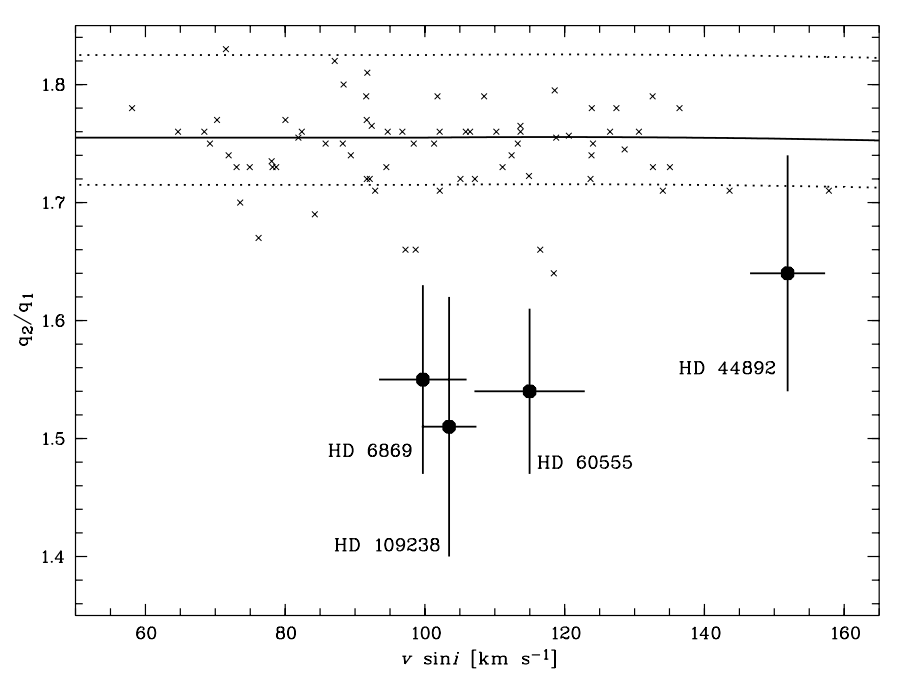

Fig. 2. Derived values of $q_{2} / q_{1}$ plotted against $v \sin i$ as derived from the first zero of the Fourier transform (with 1- $\sigma$ uncertainties). The region between dashed lines is consistent with solid body rotation for arbitrary limb darkening. For linear limb darkening with $\epsilon=0.6$, $q_{2} / q_{1}=1.76$ is expected (solid line). Crosses indicate results consistent with solid body rotation, typical errors are of the order of $\Delta q_{2} / q_{1}=0.1$ (not plotted). Four stars not consistent with $q_{2} / q_{1}=1.76$ are indicated by solid circles, error bars are plotted for them.

at the noise level for many stars, and these measurements of $q_{2}$ must be interpreted as lower limits; thus some of the measurements of $q_{2} / q_{1}$ plotted as crosses in Fig. 2 are essentially lower limits. For 74 of the 78 stars analyzed the broadening profiles are consistent with solid body rotation.

Four of our measurements are not consistent with $q_{2} / q_{1}=$ 1.76, they are indicated by full circles in Fig. 2 and errors bars are plotted for them. For three of those - HD 6869 (A9V), HD 60555 (A6V) and HD 109238 (F0IV/V) - the values of $q_{2} / q_{1}$ are significantly smaller than 1.7 . The fourth star (HD 44892 , A9/F0IV) has a value of $q_{2} / q_{1}$ marginally consistent with $q_{2} / q_{1}>1.7$ within its error bars. We will discuss this star in Sect. 3.2.

The Fourier transforms of HD 6869, HD 60555 and HD 109238 are plotted with error bars in Fig. 3. Overplotted are the Fourier transformed line profiles of stars with similar values of $v \sin i$ that are consistent with rigid rotation $\left(q_{2} / q_{1}=\right.$ 1.76). While different velocity fields, e.g., turbulence, may influence the amplitudes of the sidelobes, the zeros of the Fourier transform arise from rotational broadening (cp. Gray 1976).
One mechanism known to change the ratio $q_{2} / q_{1}$ in the manner found in HD 6869, HD 60555 and HD 109238 is solar-like differential rotation. The strength of differential rotation in terms of the parameter $\alpha$ in Eq. (1) can be calculated from $q_{2} / q_{1}$ (Reiners \& Schmitt 2003), and the respective values of $\alpha$ are given in Table 1 together with the spectral types and $v \sin i$ of the four suspected differential rotators.

\subsection{Binarity}

The spectra we used were also studied in the aim of deriving radial velocities by Grenier et al. (1999). The authors gave a "shape" flag for the correlation functions for our four candidates:

$$
\begin{array}{ll}
\text { - HD 6869: } & \text { "probable double”, } \\
\text { - HD 44 892: } & \text { "probable double", } \\
\text { - HD 60 555: } & \text { "suspected double”, } \\
\text { - HD 109238: } & \text { "probable double”. }
\end{array}
$$

Using our deconvolution method, however, we find indication for double peaks in the broadening functions of 23 of our targets, but not in the spectra of these four stars. Note that this does not mean that the 135 others are single stars since the luminous A-type stars dominate spectra of, e.g., binaries consisting of an A-type and a G-type star. The G-type spectrum will easily be hidden in the light of the A-type star. To be complete we checked the shape of the correlation function by crosscorrelating our templates with the spectra and found no indications for binarity either.

For HD 44892 and HD 109238, the literature gives hints about a single star status. There is no evidence of binarity for HD 44 892, neither in HIPPARCOS data nor in Speckle observations (Mason et al. 2001). It can be considered as single with a high level of confidence, and its spectrum is surely not affected by any significant contamination. HD 109238 is part of the sample observed by Abt \& Morrell (1995). The MK classification they derive for this object is FOV, with no suspicion of spectroscopic binarity.

For the two other objects, HD 6869 and HD 60555 two spectra are available in our data set. Individual observations of HD 6869 and HD 60555 are separated by $383 \mathrm{~d}$ and $767 \mathrm{~d}$, respectively. Inspection of the broadening functions derived from the individual spectra yields no indication of variability due to relative motions of binary components. Coadded spectra were used to derive the values of $q_{2} / q_{1}$ for both stars. Both targets 

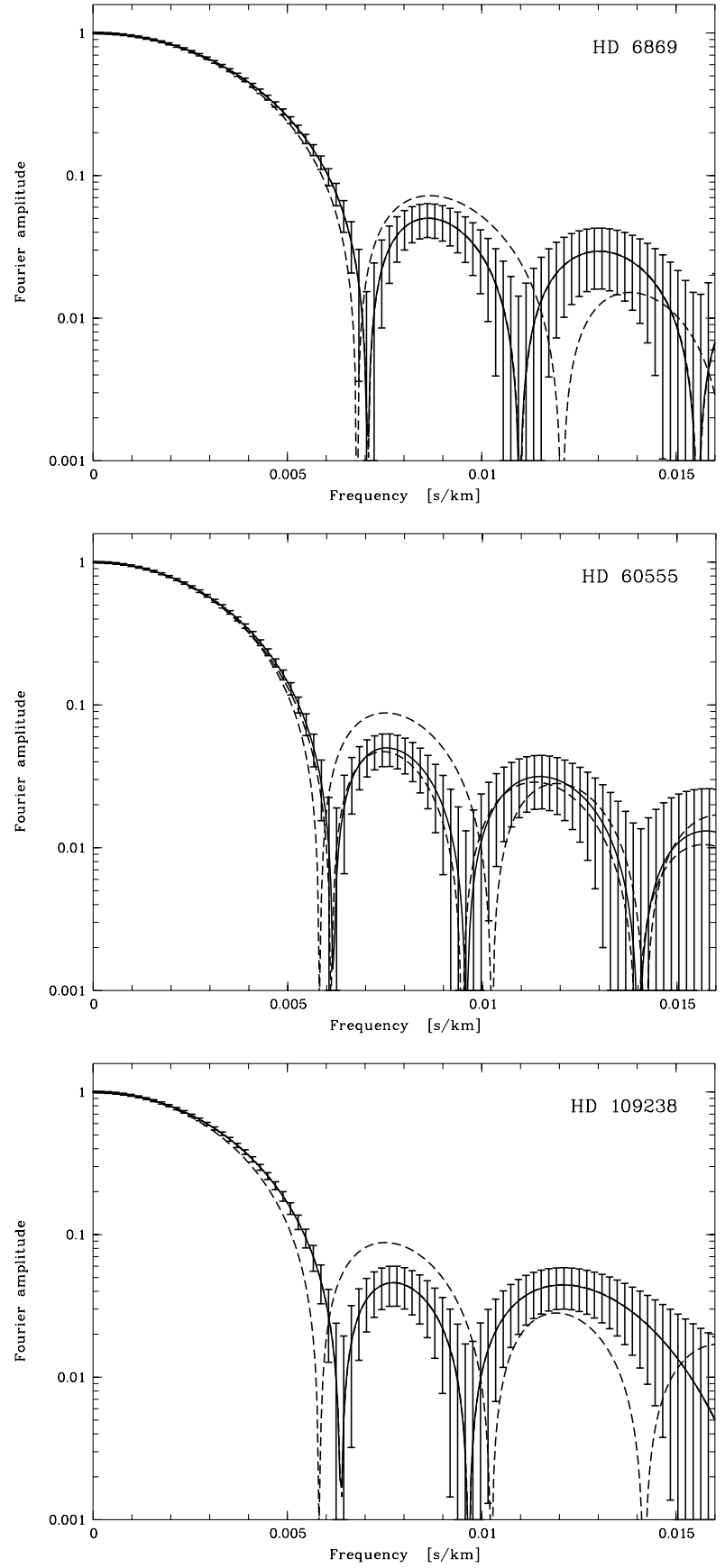

Fig. 3. Fourier transforms of HD 6869 (top panel), HD 60555 (middle), and HD 109238 (bottom panel) plotted with error bars. These stars show extremely small values of $q_{2} / q_{1}$. The spectra of HD 29920 and HD 145876 have values of $q_{2} / q_{1}$ as expected for rigid rotators and are plotted with dashed lines for comparison in the top (HD 29902), middle and bottom (both HD 145876 ) panels, respectively.

are indicated as binary stars in the literature and are discussed below.

\subsubsection{HD 6869}

HD 6869 is a binary system seen with an angular separation of about 1.'2 (ESA 1997; Horch et al. 1996). With such a low separation, compared to the width of the slit, the collected spectra are expected to be contaminated by the light of the secondary. The individual magnitudes in HIPPARCOS and TYCHO bands are given by Fabricius \& Makarov (2000) for both components and are respectively:

$$
\begin{array}{ll}
\text { - for the primary: } & H p=7.190 \pm 0.003 \mathrm{mag}, \\
& B_{\mathrm{T}}=7.42 \pm 0.01 \mathrm{mag}, \\
& V_{\mathrm{T}}=7.16 \pm 0.01 \mathrm{mag}, \\
- \text { for the secondary: } & H p=8.790 \pm 0.014 \mathrm{mag}, \\
& B_{\mathrm{T}}=9.34 \pm 0.01 \mathrm{mag}, \\
& V_{\mathrm{T}}=8.76 \pm 0.01 \mathrm{mag} .
\end{array}
$$

No significant interstellar absorption is expected (Lucke 1978) from the position of the star in the solar neighborhood $(l=$ $\left.294^{\circ}, b=-70^{\circ}, d=87 \mathrm{pc}\right)$. Considering no reddening, the temperatures of each component, derived from the $B_{\mathrm{T}}-$ $V_{\mathrm{T}}$ color index (Appendix A), are respectively $7500 \mathrm{~K}$ and $6000 \mathrm{~K}$, according to Eq. (A.1). The magnitude difference in $B_{\mathrm{T}}$ corresponds to a flux ratio of about 5.5 around $4450 \AA$, taking into account the effective temperature of both components. Given the angular separation and the large magnitude difference, the spectrum of HD 6869 can be considered as a single star spectrum of the dominating A-type star.

\subsubsection{HD 60555}

This star has been very little studied. Its spectral type in the catalogue published by Houk (1982) indicates a composite spectrum: A5/7V+(F). Grenier et al. (1999) flagged this star as variable in radial velocities from these two spectra. The ratio $E / I$ of external to internal error on radial velocity is 2.93 . The absorption spectrum of HD 60555 in the observations does not show any evidence of multiplicity, especially in the Fourier transform no indication of contamination due to the light of a secondary is apparent.

\subsection{Extremely fast rotation}

Alternative to differential rotation the shape of the broadening function and the value of $q_{2} / q_{1}$ can also be affected by very rapid rotation and gravity darkening possibly observed pole-on (cp. Reiners 2003). Flux is redistributed from the line's wings to the center when the Equator becomes cooler due to gravity darkening. As far as the lines considered are not dominated by temperature and gravity variations over the stellar surface which is the case, e.g., in the weak lines of early A-type stars as shown by Gulliver et al. (1994) - the value of $q_{2} / q_{1}$ is diminished by this effect. According to Reiners (2003), the ratio $q_{2} / q_{1}$ then only depends on the equatorial velocity $v_{\mathrm{e}}$ and on the gravity darkening law. We assume a linear gravity darkening law according to Claret (1998) and calculate the equatorial velocities $v_{\mathrm{e}, \text { rigid }}$ required to produce the measured values of $q_{2} / q_{1}$ assuming solid body rotation for the four suspected differential rotators. The results and the respective inclination angles $i$ are given in columns six and seven of Table 1 .

For HD 6869, HD 60555 and HD 109238 the values of $v_{\text {e,rigid }}$ are larger than breakup velocity; for these stars rapid solid body rotation can be ruled out as the mechanism solely responsible for the diminished ratio $q_{2} / q_{1}$. In case of HD 44892 
the rotational velocity required for the measured ratio $q_{2} / q_{1}=$ 1.64 is of the order of breakup velocity. Thus differential rotation as well as rapid solid body rotation are the two possible explanations for the measured profile shape of HD 44892.

\section{Conclusions}

We reanalyzed high quality data formerly discussed by Grenier et al. (1999) and Royer et al. (2002), with the aim of searching for differential rotation in early-type stars. With an iterative Least Squares Deconvolution method we obtained high quality broadening profiles of 158 stars with projected rotational velocities in the range $60 \mathrm{~km} \mathrm{~s}^{-1}<v \sin i<150 \mathrm{~km} \mathrm{~s}^{-1}$. We disregarded the profiles of 80 of them due to obvious asymmetries or multiplicity. For 78 stars the broadening profiles apparently reflect the rotational broadening law. Profile distortions were analyzed in terms of the ratio of the first two zeros of the Fourier transform $q_{2} / q_{1}$. Within the errors, 74 of the 78 measured profiles are consistent with the assumption of rigid rotation. Due to data quality many measurements must be considered lower limits and from this sample no conclusion can be drawn concerning values of $q_{2} / q_{1}$ possibly larger than 1.8 .

Unfortunately, for none of our sample stars interferometric measurements are available to our knowledge, e.g., Altair and Sirius have rotational velocities outside our range determined by data quality, and they are not contained in our sample.

Four stars are analyzed in detail, the profile of the A9/F0IV star HD 44892 is only marginally consistent with rigid rotation. It is likely that its profile is distorted either by differential rotation or by very rapid rotation seen pole-on; in the latter case HD 44892 would be the first star that directly shows signatures of gravity darkening in mean profile broadening as proposed by Reiners (2003). Comparison to interferometric results would be interesting especially for this star.

The broadening functions of the three stars HD 6869 (A9V), HD 60555 (A6V) and HD 109238 (F0IV/V) are not consistent with rigid - even very rapid - rotation since their equatorial velocities would be larger than breakup velocity. Although some authors suspect these stars being binaries, in our high quality spectra we find no indications of multiplicity neither in data nor in Fourier space. Since contamination due to secondaries are easily visible in Fourier space - where no sharp zeros should occur in case of the profile being a sum of two - we consider the spectra single star spectra. Differential rotation seems to be the most plausible explanation for the observed profile distortions. For these three stars the Equator is rotating about $30 \%$ faster than the polar regions. Thus we conclude that significant differential rotation seems to take place even in early-type stars not harbouring deep convection zones, the earliest object is the A6 dwarf HD 60555.

If differential rotation is the driving mechanism for stellar activity, these stars should be active, too. X-ray emission from HD 6869 was detected with the ROSAT mission and the other stars may also be X-ray sources but were simply too far away for a detection. Whether differential rotation is a common phenomenon in these stars cannot be answered by this work since only very strong differential rotation is detectable with our method. The finding of strong differential rotation among
A-type stars indicates that there is no abrupt change in rotational laws of stars around the boundary where surface convection sets in.

Acknowledgements. A.R. acknowledges financial support from Deutsche Forschungsgemeinschaft DFG-SCHM 1032/10-1. This work was partly supported by the Swiss National Science Foundation.

\section{Appendix $A: B_{T}-V_{T}$ versus $T_{\text {eff }}$}

The effective temperature can be approximated using the color index $B_{\mathrm{T}}-V_{\mathrm{T}}$. This calibration is built from the catalogue given by Cayrel de Strobel et al. (1997). They compile [Fe/H] and $T_{\text {eff }}$ determinations from the literature. These data together with TYCHO colors for these stars allow the calibration of $T_{\text {eff }}$ as a function of $B_{\mathrm{T}}-V_{\mathrm{T}}$. The resulting formula is:

$$
\begin{aligned}
\log \left(T_{\text {eff }}\right)= & 4.0132-0.87573 x+2.2194 x^{2} \\
& -5.0087 x^{3}+6.7676 x^{4}-5.1069 x^{5} \\
& +2.0638 x^{6}-0.39629 x^{7}+0.024548 x^{8},
\end{aligned}
$$

with $x=B_{\mathrm{T}}-V_{\mathrm{T}}$.

\section{References}

Abt, H. A., \& Morrell, N. I. 1995, ApJS, 99, 135

Cayrel de Strobel, G., Soubiran, C., Friel, E. D., Ralite, N., \& François, P. 1997, A\&AS, 124, 299

Claret, A. 1998, A\&AS, 131, 395

ESA 1997, The Hipparcos \& Tycho Catalogues, ESA-SP 1200

Fabricius, C., \& Makarov, V. V. 2000, A\&A, 356, 141

Gerbaldi, M., \& Mayor, M. 1989, The Messenger, 56, 12

Gray, D. F. 1976, The observation and analysis of stellar photospheres (New York: Wiley)

Gray, D. F. 1977, ApJ, 211, 198

Gray, D. F., \& Nagel, T. 1989, ApJ, 341, 421

Grenier, S., Burnage, R., Faraggiana, R., et al. 1999, A\&AS, 135, 503

Gulliver, A. F., Hill, G., \& Adelman, S. J. 1994, ApJ, 429, L81

Horch, E. P., Dinescu, D. I., Girard, T. M., van Altena, W. F., et al. 1996, AJ, 111, 1681

Houk, N. 1982, Catalogue of two-dimensional spectral types for the HD stars, vol. 3 (Michigan Spectral Survey, Ann Arbor, Dep. Astron., Univ. Michigan)

Jefferys, W. H., Fitzpatrick, M. J., \& McArthur, B. E. 1998a, Celest. Mech., 41, 39

Jefferys, W. H., Fitzpatrick, M. J., McArthur, B. E., \& McCartney, J. E. 1998b, GaussFit: A System for least squares and robust estimation, User's Manual (Dept. of Astronomy and McDonald Observatory, Austin, Texas)

Kupka, F., Piskunov, N. E., Ryabchikova, T. A., Stempels, H. C., \& Weiss, W. W. 1999, A\&AS, 138, 119

Lucke, P. B. 1978, A\&A, 64, 367

Mason, B. D., Hartkopf, W. I., Holdenried, E. R., \& Rafferty, T. J. 2001, AJ, 121, 3224

Reiners, A., \& Schmitt, J. H. M. M. 2002, A\&A, 384, 155

Reiners, A., \& Schmitt, J. H. M. M. 2003, A\&A, 398, 647

Reiners, A. 2003, A\&A, 408, 707

Renzini, A., Cacciari, C., Ulmschneider, P., \& Schmitz, F. 1977, A\&A, 61,39

Royer, F., Gerbaldi, M., Faraggiana, R., \& Gómez, A. E. 2002, A\&A, 381, 105 (RGFG)

Schmitt, J. H. M. M. 1997, A\&A, 318, 215

Wolff, S. C., Boesgaard, A. M., \& Simon, T. ApJ, 310, 360 